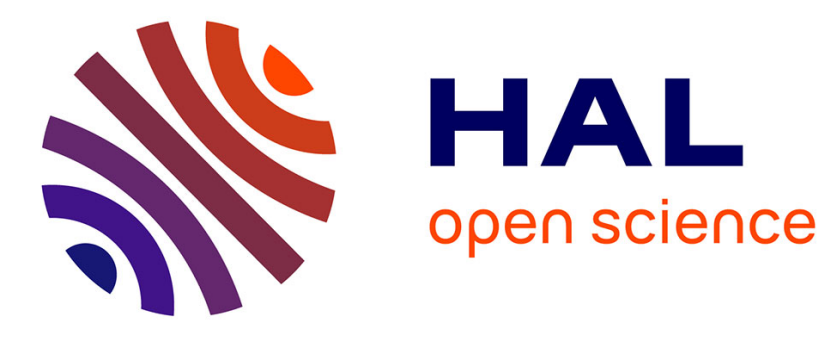

\title{
Novel method for CEP-stable seeding of few-cycle OPCPAs
}

\author{
G Cirmi, H Cankaya, P Krogen, A.-L. Calendron, Y Hua, Benoît Debord, \\ Frédéric Gérôme, Fetah Benabid, F.X Kartner
}

\section{- To cite this version:}

G Cirmi, H Cankaya, P Krogen, A.-L. Calendron, Y Hua, et al.. Novel method for CEP-stable seeding of few-cycle OPCPAs. Advanced Solid State Lasers Conference (ASSL) 2019, OSA, Sep 2019, Vienne, Austria. pp.AM2A.3. hal-02331565

\section{HAL Id: hal-02331565 \\ https://hal.science/hal-02331565}

Submitted on 20 Nov 2020

HAL is a multi-disciplinary open access archive for the deposit and dissemination of scientific research documents, whether they are published or not. The documents may come from teaching and research institutions in France or abroad, or from public or private research centers.
L'archive ouverte pluridisciplinaire HAL, est destinée au dépôt et à la diffusion de documents scientifiques de niveau recherche, publiés ou non, émanant des établissements d'enseignement et de recherche français ou étrangers, des laboratoires publics ou privés. 


\title{
Novel method for CEP-stable seeding of few-cycle OPCPAs
}

\author{
Giovanni Cirmi $^{1,2, *}$, Hüseyin Çankaya ${ }^{1,2}$, Peter Krogen $^{3}$, Anne-Laure Calendron ${ }^{1,2}$, Yi Hua ${ }^{1}$ \\ Benoit Debord $^{4}$, Frédéric Gérôme ${ }^{4}$, Fetah Benabid ${ }^{4}$ and Franz X. Kärtner ${ }^{1,2,3}$ \\ 1 Center for Free Electron Laser Science (CFEL), Deutsches Elektronen-Synchrotron (DESY) \& Department of Physics, \\ University of Hamburg, Notkestrasse 85, 22607 Hamburg, Germany \\ 2 The Hamburg Center for Ultrafast Imaging, Luruper Chaussee 149, 22761 Hamburg, Germany \\ 3 Department of Electrical Engineering and Computer Science and Research Laboratory of Electronics, \\ Massachusetts Institute of Technology (MIT), Cambridge, Massachusetts 02139, USA \\ 4 GPPMM Group, Xlim Research Institute, UMR 7252 CNRS, University of Limoges, Limoges, France \\ *giovanni.cirmi@desy.de
}

\begin{abstract}
We demonstrate a novel energy-efficient method for seeding CEP-stable OPCPAs. We couple the CEP-stable idler of a broadband OPCPA into a Kagome fiber thus compensating for its angular chirp. We show the pulse compressibility.

OCIS codes: 190.4970 Parametric oscillators and amplifiers; 060.4005 Microstructured fibers
\end{abstract}

The quest for isolated attosecond pulses (IAPs) in the water window drives the research of carrier-envelope phase (CEP) stable high-energy, short-pulse sources in the infrared. The spectral region around $2-\mu \mathrm{m}$ is a good compromise between high harmonic generation (HHG) efficiency and cut-off extension for reaching the water window spectral region. To produce few-cycle pulses at 2- $\mu \mathrm{m}$, necessary for driving IAPs, the preferred optical scheme consists in pumping optical parametric chirped pulse amplifiers (OPCPAs) with $1-\mu \mathrm{m}$ lasers. One difficulty of this approach is the generation of a few-cycle CEP-stable seed starting from a non-CEP-stable laser. Degenerate optical parametric amplifiers (OPAs) or OPCPAs with type-I $\beta$-barium borate (BBO) crystals are able to produce few-cycle signal and idler pulses, in which the idler has a stable CEP [1]. Such broadband OP(CP)As can be generated either collinearly or non-collinearly. In a collinear setup, the idler is angular-chirp free, it is spatially overlapped with the signal, has the same spectral content and the same polarization, but a different CEP. Therefore it is not possible to spatially separate the CEP-stable idler from the non-CEP-stable signal beam. In a non-collinear setup, the idler has a very strong angular chirp due to different phase matching for each signal-idler wavelength pair. In both cases, the idler cannot be used as a seed for successive amplification stages. An optical design [2,3] was introduced to address this problem, consisting in using a narrowband collinear or non-collinear OP(CP)A with signal and idler at different spectral regions with a negligible angular chirp compared with the natural beam divergence of the idler. The CEP-stable idler or its second harmonic then pumps a CEP-stable broadband supercontinuum which can be used as a seed for successive amplification stages. This method was used in several works and it is of great advantage in particular for optical parametric synthesizers due to the possibility to produce a CEP-stable seed over multiple octaves $[4,5]$.

In this work, we introduce a novel scheme which allows saving pump energy for the generation of a few-cycle CEP-stable seed for a single channel OPCPA chain. The method consists in using a broadband non-collinear OPCPA with a strongly angularly-chirped idler and focusing it into a Kagome fiber [6]. The Kagome fiber is used in a linear regime and it homogenizes the wave-vector directions of all idler wavelengths, similarly to the technique used for homogenizing diode lasers [7]. At the output of the fiber, the CEP-stable broadband idler has a well-defined propagation direction, i.e. no angular chirp, and can be directly used to seed the following amplification stages. We believe that our technique is simpler than other techniques developed to compensate for the idler angular chirp of an OPA $[8,9]$.

In our setup (Fig. 1a) we utilized $308 \mu \mathrm{J}$ of total pump energy from the same home-built Yb:KYW regenerative amplifier [10] used in [5] and built a 2-stage degenerate OPCPA with BBO crystals. The idler output energy of the second OPCPA stage is $10.6 \mu \mathrm{J}$ and it has a strong angular chirp which we measured to be $\sim 3.6^{\circ}$. We focused the idler into a Kagome fiber with $\sim 22-\mathrm{cm}$ length (7-cell core design with 63 core diameter). The fiber output energy is $3.8 \mu \mathrm{J}$, which can be further improved by optimizing the coupling efficiency (currently $36 \%$ ) into the Kagome fiber. The spectra of the idler before and after the Kagome fiber are shown in Fig. 1 (b). The fiber output has no residual detectable angular chirp. 

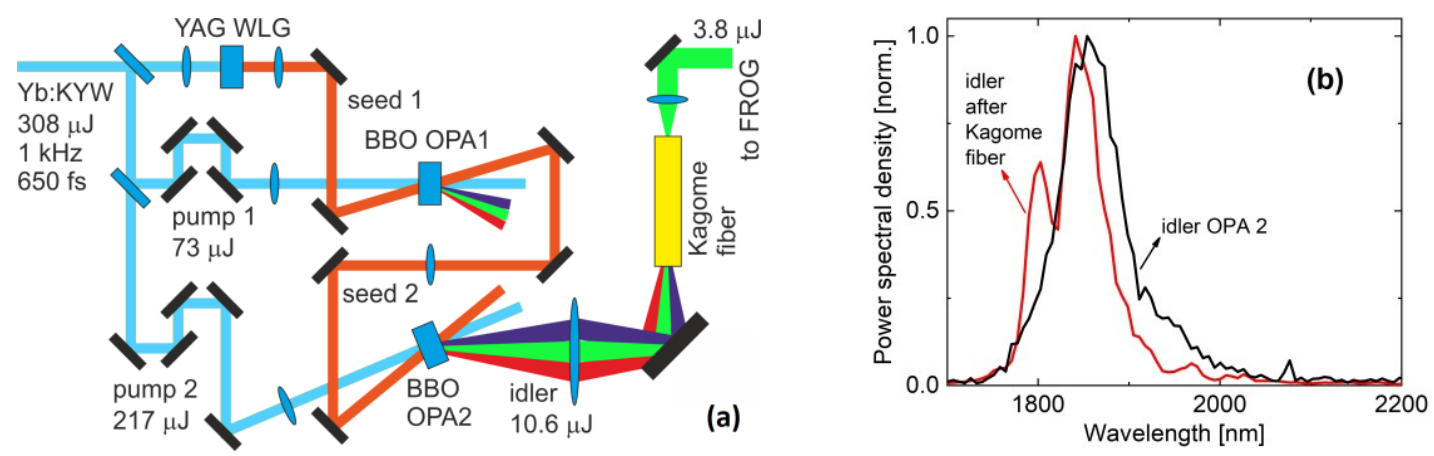

Fig. 1. (a) Optical layout. A 10-mm YAG was used for white light generation (WLG), and 2 consecutive 4mm BBO crystals were used for the 2 OPA stages (b) Spectra of the idler of the second OPA stage before and after the Kagome fiber.

To demonstrate the temporal compressibility of our pulses, we performed second harmonic generation frequency resolved optical gating (SHG FROG) measurements and retrieved 60-fs pulse duration. As shown in Fig. 2, the retrieved phase is smooth and we expect that it can be easily compressed to 30 -fs transform limit.
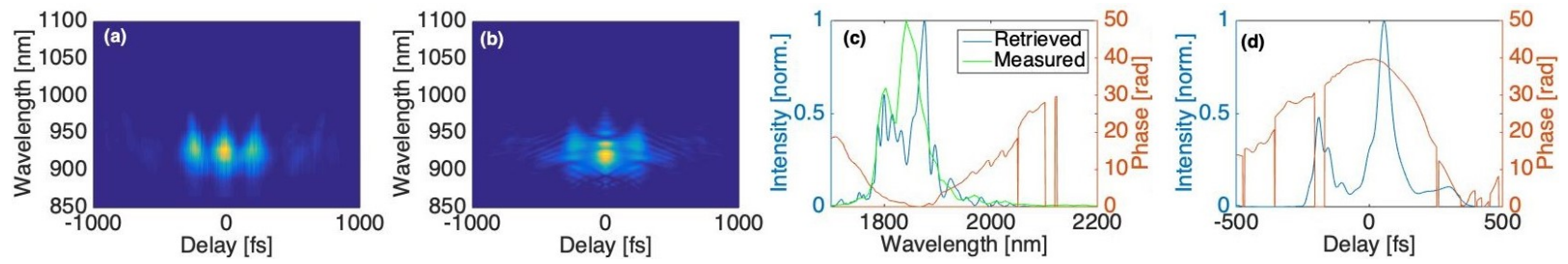

Fig. 2. Measured (a) and retrieved (b) FROG traces; (c) measured (green) and retrieved (blue) spectral intensity; retrieved spectral phase (orange); (d) retrieved temporal intensity (blue) and phase (orange).

[1] A. Baltuška, T. Fuji, and T. Kobayashi, "Controlling the Carrier-Envelope Phase of Ultrashort Light Pulses with Optical Parametric Amplifiers” Phys. Rev. Lett. 88, 133901 (2002).

[2] C. Manzoni et al., "Tunable few-optical-cycle pulses with passive carrier-envelope phase stabilization from an optical parametric amplifier", Appl. Phys. Lett. 90, 171111 (2007).

[3] G. Cirmi et al., “Carrier-envelope phase stable, few-optical-cycle pulses tunable from visible to near IR”, JOSA B 25, B62 (2008).

[4] O. D. Mücke et al., "Toward Waveform Nonlinear Optics Using Multimillijoule Sub-Cycle Waveform Synthesizers", JSTQE 21, 8700712 (2015).

[5] H. Çankaya et al., "40- $\mu \mathrm{J}$ passively CEP-stable seed source for ytterbium-based high-energy optical waveform synthesizers", Opt. Expr. 24, 25169 (2016).

[6] K. Murari et al., "Kagome-fiber-based pulse compression of mid-infrared picosecond pulses from a Ho:YLF amplifier", Optica 3, 816 (2016).

[7] M. Traub et al., "Homogenization of high power diode laser beams for pumping and direct applications", Proceedings 6104, High-Power Diode Laser Technology and Applications IV; 61040Q (2006).

[8] K. Okamura and T. Kobayashi, "Octave-spanning carrier-envelope phase stabilized visible pulse with sub-3-fs pulse duration", Opt. Lett. 36, 226 (2011).

[9] S.-W. Huang, J. Moses, and F. X. Kärtner, "Broadband noncollinear optical parametric amplification without angularly dispersed idler", Opt. Lett. 37, 2796 (2012).

[10] A.-L. Calendron, H. Çankaya, and F. X. Kärtner, "High-energy kHz Yb:KYW dual-crystal regenerative amplifier”, Opt. Expr. 22, 24752 (2014). 